\title{
Testing Competing Claims About Overclaiming
}

\author{
Goecke, B. ${ }^{1}$, Weiss, S. ${ }^{1}$, Steger, D. ${ }^{1}$, Schroeders, U. ${ }^{2}$, \& Wilhelm, O. ${ }^{1}$ \\ ${ }^{1}$ Ulm University, Ulm, Germany \\ ${ }^{2}$ University of Kassel, Kassel, Germany
}

\begin{abstract}
Author Note:
Correspondence concerning this article should be addressed to Benjamin Goecke, Institute for Psychology and Education, Ulm University, Albert-Einstein-Allee 47, 89081 Ulm, Germany. Telephone: +49-(0)731/50 25374.

E-mail: Benjamin.Goecke@uni-ulm.de
\end{abstract}

The data is available along with syntax for the statistical analysis at: https://osf.io/4bnv5/

This manuscript has been accepted for publication in Intelligence in its current form. This final manuscript version accepted for publication may not exactly replicate the final version published in the journal. It is not the copy of record.

The published version can be found under: https://doi.org/10.1016/j.intell.2020.101470

Goecke, B., Weiss, S., Steger, D., Schroeders, U., \& Wilhelm, O. (2020). Testing competing claims about overclaiming. Intelligence, 81, 101470.

(C) This manuscript version is made available under the CC-BY-NC-ND 4.0 licence. 


\begin{abstract}
Overclaiming has been described as people's tendency to overestimate their cognitive abilities in general and their knowledge in particular. We discuss four different perspectives on the phenomenon of overclaiming that have been proposed in the research literature: Overclaiming as a result of a) self-enhancement tendencies, b) as a cognitive bias (e.g., hindsight bias, memory bias), c) as proxy for cognitive abilities, and d) as sign of creative engagement.
\end{abstract} Moreover, we discuss two different scoring methods for an OCQ (signal detection theory vs. familiarity ratings). To distinguish between the different viewpoints of what overclaiming is, we juxtaposed overclaiming, as indicated by claiming familiarity with non-existent terms, with fluid and crystallized intelligence, self-reported knowledge, creativity, faking ability, and personality. Overclaiming was measured with a newly comprised overclaiming questionnaire. Results of several latent variable analyses based upon a multivariate study with 298 participants were: First, overclaiming is neither predicted by honesty-humility nor faking ability and therefore reflects something different than mere self-enhancement tendencies. Second, overclaiming is not predicted by crystallized intelligence, but is highly predictive of self-reported knowledge and, thus, not suitable as an index or a proxy for cognitive abilities. Finally, overclaiming is neither related to divergent thinking and originality, and only moderately predicted by self-reported openness creativity from the HEXACO which means that overclaiming does not reflect creative ability. In sum, our results favor an interpretation of overclaiming as a phenomenon that requires more than self-enhancement motivation, in contrast to the claim that was initially proposed in the literature.

Keywords: overclaiming, declarative knowledge, self-reported knowledge, creativity, intelligence, faking 
Testing Competing Claims About Overclaiming

\section{Introduction}

What do you know about the Higgs-Boson? How much do you know about plate tectonics? And what about cholarine? Depending on your knowledge on physical sciences, you may or may not be familiar with the first two topics - the Higgs-Boson and plate tectonics. However, it is impossible that you know anything about cholarine, simply because it does not exist. It is one of the fictitious words (or foils) used in an Overclaiming Questionnaire (OCQ; Paulhus et al., 2003). The phenomenon that people-intentionally or not - overestimate their knowledge of terms, events, concepts, people, etc. that cannot be known because they do not exist is called overclaiming (e.g., Paulhus et al., 2003; Phillips \& Clancy, 1972).

Four competing theoretical perspectives on overclaiming can be distinguished. First, Paulhus and colleagues (2003, p. 899) understood overclaiming as a "trait-like tendency to self-enhance”. Second, overclaiming can be seen as a cognitive processing bias (Müller \& Moshagen, 2018; Paulhus, 2011). Third, accurately distinguishing between existent and nonexistent words on OCQs might be taken as a proxy for declarative knowledge since persons with more knowledge might overclaim less (Paulhus \& Harms, 2004). Finally, overclaiming might occur whenever people are willing to encounter, engage with, or elaborate on new concepts or ideas (Dunlop et al., 2017), possibly making overclaiming akin to creativity. Regardless of which perspective one adopts, many studies placed overclaiming into a broader nomological network including faking and cheating (Bing et al., 2011; Feeney \& Goffin, 2015; Kam et al., 2015; Steger et al., 2019a), fluid and crystallized intelligence (Hülür et al., 2011; Paulhus \& Harms, 2004), and the narcissism facet of dark personality (Grosz et al., 2017; Paulhus et al., 2003; Williams et al., 2002). Although overclaiming questionnaires are a frequently used instrument in several disciplines of psychology, our understanding of their underlying nature is still limited. 
In the current study, we examine overclaiming as indicated by claiming familiarity with non-existent terms (Phillips \& Clancy, 1972) in light of the above-mentioned perspectives by deriving predictions from all viewpoints and testing them within a latentvariable approach. We want to shed light on the relation of overclaiming with declarative knowledge, self-reported knowledge, creative ability, honesty-humility and openness creativity, faking ability, and fluid intelligence.

\section{Scoring Methods of Overclaiming: Raw Responses vs. Signal Detection Indices}

A number of researchers worked on different approaches to assess (e.g., Ackerman \& Ellingsen, 2014; Ziegler et al., 2013) and score overclaiming (Hülür et al., 2011; Paulhus \& Petrusic, 2007). The basic idea of using foils for studying familiarity claims can be traced back to Raubenheimer (1925), who laid the foundation of the Overclaiming Technique (OCT). Afterwards, this method had long been forgotten until it was revived in consumer behavior research (Phillips \& Clancy, 1972). The OCT finally made its way to individual differences research in an attempt to use the technique for studying self-enhancement tendencies independent of ability (Paulhus et al., 2003). In the original OCQ (OCQ-150; Paulhus et al., 2003), participants were asked to rate their familiarity with 150 items by rating them on a scale ranging from "never heard of it" to "know it very well". Since then, the OCT and a variety of OCQs were developed and used in psychological research (e.g., Atir et al., 2015; Dunlop et al., 2017, 2019; Mesmer-Magnus et al., 2006; Pennycook \& Rand, 2019; Ziegler et al., 2013). Although alternative assessment methods of overclaiming are interesting, we will not further discuss them here and will instead focus on competing scoring methods of conventional OCQs.

With respect to the methodology tied to the OCT, it is necessary to distinguish several terms and statistical indices. An OCQ consists of two different types of items, namely reals and foils. Whereas reals refer to items that are existent terms, concepts, or words, foils are non-existent as terms, concepts, or words. Thus, reals and foils belong to the item-side of an 
OCQ. Based on participants' answers to these items, several person indices can be computed. On the one hand, the familiarity rating of reals and foils may be read precisely as such: as ordinary raw responses on a rating scale for familiarity. Asking participants to rate their familiarity with existent terms, concepts, or words, probes their self-reported knowledge (e.g., Bing et al., 2011; Fell et al., 2019; Hülür et al., 2011), whereas familiarity ratings with items that cannot be familiar indicate overclaiming (Bing et al., 2011; Randall \& Fernandes, 1991; Phillips \& Clancy, 1972). This scoring of an OCQ is straightforward and economical, as it only requires the independent computation of mean familiarity ratings for reals and foils to express a person's standing on self-reported knowledge and overclaiming, respectively.

On the other hand, a signal detection approach is commonly used and often recommended to analyze OCQ data (e.g., Paulhus, 2011; Paulhus et al., 2003). The two most prominent indices that are computed within the framework of signal detection are the accuracy index $d^{\prime}$ (d prime, also called $O C$ accuracy) and the index for response bias $c$ (criterion location, also called $O C$ bias). $d$ ' is thought to be indicative of a person's sensitivity to accurately categorize reals as existing and foils as non-existing; $c$ can be understood as an indicator of a person's “yes-tendency", indicating a general tendency to claim familiarity with the items of an OCQ (MacMillan \& Creelman, 2005; Paulhus et al., 2003).

A number of approaches have been developed to obtain both scores (e.g., Stanislaw \& Todorov, 1999; Wixted, 2020). All approaches have in common that $d$ ' and $c$ rely on the same information for computation, namely the hit rate and the false alarm rate from a binary classification table. The hit rate is usually computed as the proportion of correct familiarity ratings for reals and the false alarm rate is computed as the proportion of false familiarity ratings for foils. Importantly, $d$ ' and $c$ rely on ratings for both reals and foils and are logically and computationally dependent. This is problematic in a statistical sense, because psychometrically the accuracy index should be unaffected by the response bias to be interpretable. Yet, $d$ ' is only unaffected by $c$ if the distributions of familiarity rating of signal 
trials (which translates to the hit rate) and familiarity ratings of noise trials (which corresponds to the false alarm rate) are normally distributed, have equal variances (Kellen $\&$ Klauer, 2018; Stanislaw \& Todorov, 1999), and are uncorrelated. However, these assumptions are usually not met (e.g., DeCarlo, 2010; MacMillan \& Creelman, 2005; Mickes et al., 2007; Selker et al., 2019; Stanislaw \& Todorov, 1999; Starns \& Ratcliff, 2014; Swets, 1986).

Besides these statistical considerations, both indices are difficult to grasp in terms of the underlying cognitive processes and the conceptual link between manifest indicators and latent constructs. While the interpretation of responses to reals and foils as familiarity ratings in a latent variable approach is straightforward, this interpretation is not clear in signal detection theory. Two processes - the correct identification of reals and the correct rejection of foils - are intertwined, so that a lower score in OC bias can be achieved by a better hit rate, a lower false alarm rate, or both.

Taken together, indices of signal detection theory are more problematic and less straightforward indicators to exhaust responses from an overclaiming questionnaire than commonly assumed. The $d$ ' and $c$ indices are confounded by virtue of being computed based on the same underlying information (hit rate and false alarm rate) and the information captured in ratings does not meet statistical assumptions for computing adequate person estimates for bias and accuracy. Due to these reasons, we do not pursue the frequently used signal detection scoring approach and rather rely on the information provided by mean familiarity ratings of reals and foils, respectively. We use these ratings as manifest variables in a structural equation model to examine individual differences in self-reported knowledge and overclaiming. However, we acknowledge the widespread use of signal detection analysis for OCQs and therefore provide a set of results and a description of the corresponding formulas (see supplementary material: SM Figure 1, SM Table 2, SM Table 3).

\section{What Drives Overclaiming Behavior}


In the following, we discuss four theoretical perspectives on the phenomenon of overclaiming as measured by an OCQ. That is, (a) overclaiming reflects self-enhancement tendencies, (b) overclaiming is a product of a cognitive processing bias, (c) rating familiarity for reals and foils on an OCQ is an index of cognitive abilities, and (d) overclaiming is a type of creative engagement. An overview of the theoretically-derived predictions made by the four theoretical accounts is given in Table 1 for the above-discussed mean familiarity ratings for reals (SRK) and foils (OC) on the one hand, and for the conventional signal detection indices (OC bias and OC accuracy) on the other hand.

\section{Table 1}

Synopsis of Theoretical Perspectives on Overclaiming and Corresponding Predictions.

\begin{tabular}{rcccc}
\hline Index & $\begin{array}{c}\text { Overclaiming } \\
(\text { OC })\end{array}$ & $\begin{array}{c}\text { Self-Reported } \\
\text { Knowledge } \\
(\text { SRK })\end{array}$ & $\begin{array}{c}\text { OC bias } \\
(c)\end{array}$ & $\begin{array}{c}\text { OC accuracy } \\
\left(d^{\prime}\right)\end{array}$ \\
\hline $\begin{array}{c}\text { If responses on OCQ } \\
\text { are influenced by... }\end{array}$ & & & + & - \\
\hline $\begin{array}{r}\text { Self-enhancement } \\
\text { tendencies }\end{array}$ & + & + & + & - \\
$\begin{array}{r}\text { Cognitive Biases } \\
\text { (e.g., hindsight bias, } \\
\text { memory bias) }\end{array}$ & + & + & & + \\
$\begin{array}{r}\text { Cognitive Abilities } \\
\text { (i.e., knowledge) }\end{array}$ & - & + & - & - \\
Creative Engagement & + & + & + & + \\
\hline Note. The table describes which index of an OCQ should show a positive $(+)$ or negative $(-)$
\end{tabular}

relation to constructs that are reflective for the theoretical assumption in question (e.g., a $g c$ measure for the perspective that an OCQ is reflective for cognitive abilities). OC and SRK are based on familiarity ratings of foils and reals, respectively. OC bias and OC accuracy are both calculated based on the hit rate and the false alarm rate.

From the first perspective, overclaiming behavior is understood as reflecting a overarching self-enhancement tendency, which describes the tendency to present oneself in a desirable way (e.g., when completing self-reports). In line with this notion, Paulhus and 
colleagues (2003) found convergent validity of the OC bias index with established measures of self-enhancement such as the Narcissistic Personality Inventory $(r=.35$; Raskin \& Hall, 1979) and the Self-Deceptive Enhancement Scale ( $r=.30$; Paulhus \& Reid, 1991). Paulhus and colleagues (2003) concluded that the OCT is useful to assess the proneness to selfenhance, which would be a useful instrument in various contexts that rely on self-report measures (e.g., personnel selection), as these frequently suffer from various biases (Van Vaerenbergh \& Thomas, 2013; Ziegler, 2015). Generally, engaging in overclaiming has been described as a potentially economical marker for positivity biases or self-enhancement tendencies (e.g, Bensch et al., 2017; Dunlop et al., 2019; Paulhus et al., 2003). It has been considered as a type of remedy and an efficient mean of assessing individual differences in the tendency to systematically misrepresent oneself in self-reports (Ludeke \& Makransky, 2016; Paulhus et al., 2003; Paulhus \& Harms, 2004). In this sense, overclaiming might reflect people's tendency to present themselves in a socially desirable way, that is, to intentionally exaggerate self-reports on desirable attributes, which is also termed positive impression management (Paulhus, 1984; Paulhus \& Reid, 1991). In the same vein, subjects may claim to have more knowledge than they actually possess because they unintentionally overstate or overrate their abilities. This form of overclaiming might be labeled self-deceptive enhancement (Paulhus \& Reid, 1991). The distinction between intentionally and unintentionally overclaiming is interesting, as scales measuring impression management and self-deceptive enhancement have been found to be almost unrelated (Paulhus, 2002), indicating a substantial difference between consciously and subconsciously misrepresenting oneself. Accordingly, overclaiming could be seen as a way to positively misrepresent oneself, which puts the concept in relation to the personality factor of honesty-humility (Ashton \& Lee, 2001), as well as faking (i.e., faking good, Ziegler, 2011). Although both honestyhumility and faking are not direct measures of self-enhancement, overclaiming, honestyhumility, and faking share the idea of a persons' misrepresentation. However, faking and 
dishonesty is more of a motivated deception, while self-enhancement overlaps with positive self-beliefs such as self-esteem and confidence.

Regarding the empirical evidence, impression management could be predicted by honesty-humility (de Vries et al., 2014). This relationship makes sense, because describing oneself (deliberately) in a more positive manner should correspond to being less honest and less humble. The same logic can be applied to the association of overclaiming with honestyhumility, although recent evidence shows no relation between different indices of overclaiming and honesty-humility (OC bias: Dunlop et al., 2019; Müller \& Moshagen, 2019; mean familiarity ratings of foils: Steger et al., 2020), thus suggesting overclaiming corresponds to unintentionally overrating one's abilities.

Apart from honesty-humility, faking was repeatedly described as a deliberate positive self-presentation or an intentional bias (e.g., Dunlop et al., 2019; Geiger et al., 2018; Ziegler, 2011), and the degree to which it met requirements should be considered an ability (Geiger et al., 2018). In line with this idea and given theoretical accounts of faking ability (e.g., Ziegler, 2011), it should be considered that the setting in which an OCQ is administered (low-stakes vs. high-stakes) might play an important role for the validity of overclaiming as a potential marker of deliberate positive self-representations such as faking (Dunlop et al., 2019). More precisely, the evidence that the OC bias index is predictive of faking in low-stakes settings is rather weak (Dunlop et al., 2019; Feeney \& Goffin, 2015; Ludeke \& Makransky, 2016; Müller \& Moshagen, 2019), higher in high-stakes setting (e.g., Bing et al., 2011; Dunlop et al., 2019), but still not ubiquitous (Bensch et al., 2019).

From the second perspective, engaging overclaiming can also be seen as a sort of cognitive processing bias. It was argued that due to the design of the OCQ — around 20\% foils embedded in about $80 \%$ reals — the familiarity of an item might be overrated, resulting in a memory bias (Müller \& Moshagen, 2018; Paulhus, 2011). That is, foils are typically produced and presented in a manner that makes them plausible and sometimes even orthographically or 
linguistically similar to reals (Ackerman \& Ellingsen, 2014). To this end, the item construction rationale of an OCQ might lead to increased processing fluency within persons while evaluating foils. Due to their resemblance with real words, a false feeling of familiarity can lead to a false feeling of knowing (Müller \& Moshagen, 2018). In turn, this accessibility would result in a high familiarity rating of non-existing items (Dunlop et al., 2017). This kind of spurious sense-making and the apparent misattribution of fluency for real familiarity are processes that are also linked to reconstruction biases of the well-known hindsight bias, which has been shown to explain some of the variance in a variant of the OC bias parameter (Müller \& Moshagen, 2018). This perspective contributes to the understanding of overclaiming by emphasizing the subconscious rather than the conscious account of it. However, contrary to seeing overclaiming as subconscious and self-deceptive behavior, the explanation for overclaiming from this point of view focuses on issues emerging from the typical method of measurement, namely questionnaires, and its elements, namely single items. That is, overclaiming occurs inadvertently, due to shallow characteristics of foils, and not due to any self-enhancement motivations.

Third, overclaiming has also been juxtaposed with several cognitive abilities such as fluid intelligence $(g f)$ and crystallized intelligence $(g c)$-both self-reported and objectively measured. People generally tend to overestimate their own abilities in self-reports, and their own knowledge in particular (Kruger \& Dunning, 1999). Correlations between self-reported abilities and objective tests of cognitive abilities are only moderate, even when persons rating themselves received training in self-estimation (Jacobs \& Roodenburg, 2014). This finding is further supported by meta-analytically estimated correlations between self-reports and objective tests of cognitive abilities, which are also only moderate $(r=.33$, Freund $\&$ Kasten, 2012; $\rho=.29$, Mabe \& West, 1982), demonstrating a mismatch between persons' selfperceived and actual abilities. Nonetheless, it has been argued that the OC accuracy index might be used as a proxy for $g c$ (Paulhus \& Harms, 2004), despite the fact that the moderate 
correlations between the OC accuracy index and $g c$-varying between $r=.24$ and .50 (Bensch et al., 2017; Hülür et al., 2011, 2014; Paulhus \& Harms, 2004; Ziegler, Kemper, \& Rammstedt, 2013) — have raised doubts about this claim. In sum, the signal detection scored familiarity ratings (i.e., OC accuracy) on OCQs were described as a "practical measure of global cognitive ability" ( $\beta=.51$; Paulhus $\&$ Harms, 2004, p. 311), but the empirical evidence paints a more nuanced picture.

Finally, Hülür and colleagues $(2011 ; 2014)$ linked overclaiming with intellectual investment traits such as typical intellectual engagement, self-motivated cognition, and the personality facet of openness to new ideas. Given the strong conceptual and empirical overlap between these concepts (Mussel, 2013), one could argue that overclaiming behavior is a byproduct of open-minded people who value new ideas, concepts, and terms; these people tend to overrate their familiarity with novel items. This perspective is supported by results showing that typical intellectual engagement and openness - as measured by the five-factor model (McCrae \& Costa, 1989) — are positively related to overclaiming (Ackerman \& Ellingsen, 2014). Such results might indicate that subjects scoring higher in openness encounter unknown terms in a less skeptical or rational manner but are willing to develop ad hoc ideas of what an unfamiliar term might mean. In the alternative six-factor model of personality (HEXACO; Ashton \& Lee, 2001), the openness factor (originally termed intellect/imagination) includes the facets of aesthetic appreciation, inquisitiveness, creativity, and unconventionality. Both openness factors (from the HEXACO and the five-factor model) are highly correlated and reported as mostly congruent ( $r=.71$; Moshagen et al., 2014). Especially, the openness factor based on the HEXACO includes items that might trigger endorsement from participants who feel an attraction towards foils, because these items emphasize the experience of the unknown. This idea is supported by medium correlations between OC bias and openness in the HEXACO taxonomy ( $r=.34$, Dunlop et al., 2017). Findings relating short scales of the openness factor based on the five-factor model with OC 
bias were inconclusive ( $r=.02$, Bensch et al., 2017; $r=.25$, Williams et al., 2002). The HEXACO factor of openness has also been predictive of creativity $(\beta=.55$; Silvia et al., 2011), for the reason that more creative persons may be prone to overclaim as they are more willing to encounter, engage in, and elaborate on unknown and unfamiliar ideas, concepts, or terms. Bearing these considerations in mind, examining the relationship among the creativity facet of openness (as proposed by the HEXACO), creativity as an ability, and overclaiming might further our understanding on what possibly drives overclaiming behavior.

\section{Research Aims}

Given the competing perspectives on overclaiming and its potential utility for practitioners, it seems pivotal to examine overclaiming more broadly within a comprehensive nomological network in order to further elaborate on its very nature. The essential goal of our study was to provide a test of the accounts outlined above that understand overclaiming as an interesting phenomenon that is - from our point of view - driven by more than a cognitive processing bias and should therefore be considered from a variety of viewpoints.

a. First, we present a measurement model of overclaiming and self-reported knowledge based on mean familiarity ratings.

b. Then, according to the first perspective that overclaiming behavior is part of the nomological net of a persons' tendency to misrepresent, we test the prediction that overclaiming and self-reported knowledge are partially accounted for by honesty-humility and faking ability, because the conscious tendency to positively misrepresent oneself (as presumably measured with the OCQ) should correspond to individual differences in honesty-humility and faking ability—where less honest/humble individuals engage more in overclaiming, and individuals better in faking also tend to overclaim more.

c. In addition, concerning the idea that overclaiming behavior might reflect cognitive abilities, we examine the amount of variance in overclaiming and 
self-reported knowledge that could be explained by $g c$ and $g f$ (see also Hülür et al., 2011).

d. Finally, we try to put the fourth perspective to a critical test. We do so by regressing individual differences in overclaiming and self-reported knowledge on individual differences in creative abilities and the creativity items of the openness scale. According to the fourth perspective, a substantial amount of variance in overclaiming and self-reported knowledge should be explainable by creativity (i.e., divergent thinking and originality) and openness (depicted by creativity indicators).

\section{Method}

\section{Participants and Procedure}

The current examination was part of a larger, multi-center study assessing creativity (Weiss et al., 2020). The analyses rely on a subsample of 298 participants after data cleaning (for more information see Weiss and colleagues (2020)). The participants completed seven hours of (mostly) computerized testing and were financially compensated. Five hours of testing were done in a laboratory setting, while nearly two hours of testing could be done from home. All participants provided informed consent for the study and a local ethics committee approved the data collection. The majority of the participants reported German as their first language $(N=280)$ and had a high school diploma $(N=278)$. Non-native speakers were sufficiently fluent in German to complete all measures. Seventy-two percent of the sample were female; mean age was $M=24.5$ years $(S D=5.1)$. We restrict our description of indicators to the measures pertinent to the present research questions.

\section{Measures}

\section{Overclaiming Questionnaire}

We used a newly-compiled set of 149 items to measure overclaiming with 120 reals and 29 foils in five broad domains (Natural Sciences, Life Sciences, Social Sciences, 
Humanities, and Pop Culture), in line with findings on the dimensionality of knowledge (Steger et al., 2019). All items were selected based on an overall collection of $n=1,033$ items (partly developed by the authors, partly based on Hülür et al., 2011) that covered a broad range of content, for example, for natural sciences items about physics, chemistry, computer science. Prior to item selection, 12 raters evaluated the quality and the dissimilarity of the foils to existing words as well as the difficulty of the reals for half of the items each. The quality of foils was rated on a scale from one (low quality) to five (high quality) based on their surface similarity towards reals (e.g., low similarity in spelling indicates a high quality) and their believeability (e.g., poor plausibility indicated low quality). The difficulty of reals was rated in years of education (on a scale from one to 20, including tertiary education) necessary to solve the item with a probability of .80 . For the final item set, we selected reals and foils with high difficulties and of sufficient quality. The aggregated data of these ratings for the selected item set, as well as the original mean familiarity ratings of the items that were selected from the OCQ of Hülür and colleagues (2011) are presented in the supplementary material (SM Table 1). Regarding the response scale of the OCQ, we chose a format with fully-labeled response options (e.g., Weng, 2004) that has been successfully used in the past (Hülür et al., 2011). Participants had to indicate their familiarity to words on a 5-point scale $(1=$ Never heard of it before, $2=$ Heard of it before, but cannot describe it exactly, $3=I$ could describe it roughly, $4=I$ could describe it relatively precise, and $5=I$ could give an exact description). For the complete questionnaire, see the online repository.

\section{Fluid and Crystallized Intelligence}

$G f$ was measured with the verbal and the figural subtests of the Berlin Test of Fluid and Crystallized Intelligence (BEFKI; Wilhelm et al., 2014). The verbal aspect of $g f$ was assessed via relational reasoning, that is, participants had to derive logically-valid conclusions based on a set of given premises. The figural aspect of $g f$ was composed of a sequence of geometric drawings that changed their shading and form according to certain rules. 
Participants had to infer these rules and to select missing drawings out of several response alternatives. Two items of the verbal scale had to be removed from further analysis due to extreme difficulties. $G c$ was measured with two parallel test forms compiled of 102 randomly-assigned items each from a larger pool of declarative knowledge items (Steger et al., 2019, 2020). Also, the broad content domains of $g c$ matched the domains of the overclaiming questionnaire.

\section{Creativity}

The test battery included a variety of verbal and figural divergent thinking tests (Carroll, 1993), as they are used for the measurement of creative ability along two domains: fluency (quantity of results in a given time) and originality (quality of results). The battery included four retrieval fluency tests: the similar attributes (SA including six items) and the inventing names (IN including 18 items) tests were both adapted from the "Verbaler Kreativitätstest” [verbal creativity test] (VKT; Schoppe, 1975), while the retrieval fluency (RF including six items) and the figural fluency (FF including four items) test were used from the Berlin Structure-of-Intelligence test (Jäger et al., 1997). Originality was assessed with two tests: combining objects (CO, including 12 items) (French et al., 1963) and inventing nicknames (NI, including nine items) (VKT; Schoppe, 1975). Divergent thinking items are usually open-ended and require human rating. All answers were counted (fluency) or scored (originality) by three independent human raters. The originality tests were coded regarding the creativity of an answer on a five-point Likert scale (Amabile, 1982; Silvia et al., 2008), resulting in good inter-rater reliability (Weiss et al., 2020). The measurement model was specified with test-scores (i.e., mean across all rated items) for each of the mentioned tests as indicators. Two creativity tests measuring originality load on a nested factor, in addition to an overarching divergent thinking factor. For a more detailed description of the tests, their psychometric properties, and the measurement model of divergent thinking, please see Weiss and colleagues (2020). 


\section{Personality}

Personality was measured with the German 60-item version of the HEXACO (Moshagen et al., 2014), covering the personality factors of Honesty-Humility, Emotional Stability, Extraversion, Agreeableness, Conscientiousness, and Openness, with 10 items each. Participants had to indicate their agreement with statements on a five-point scale ranging from "strongly disagree" to "strongly agree" (e.g., "I'm interested in learning about the history and politics of other countries” for openness). For modeling honesty-humility (see Figure 2), we used three parcels with similar mean values. Based on the theoretical considerations in the introduction, the openness factor in Figure 4 only includes the three items of the openness scale that tap creativity (Ashton \& Lee, 2009).

\section{Faking}

Faking ability was measured using the paradigm presented in Geiger, Olderbak, Sauter, and Wilhelm (2018) with items from the Work Style Questionnaire (WSQ), a selfreport measure originally developed for O*NET (Borman et al., 1999). The questionnaire consists of 16 items defining various aspects of a typical work style (e.g. Achievement/Effort: "Establishing and maintaining personally challenging achievement goals and exerting effort towards mastering tasks"). Participants were asked to select a response option from a fivepoint Likert scale ranging from "Not at all like me" to "Extremely like me" for all 16 items for five different jobs with distinct work style patterns (pilot, talk show host, software engineer, tour guide, insurance seller). They were instructed to select response options in a way to maximize their chances of being hired for the five jobs in a personality task-style fashion ("Please rate how much this characteristic describes you"). For scoring, we applied the profile similarity metric shape as reported in Geiger and colleagues (2018), i.e. the participants' responses were correlated to expert ratings of the jobs work styles from O*NET. Slightly alienating from Geiger and colleagues (2018), we rounded the optimal profile scores (rational numbers) in order to compute Spearman rank-order correlations per participant, due 
to administering a discrete five-point Likert scale (natural numbers) in our study. These correlations were used as indicators for a general latent model of faking (Geiger et al., 2018). For validation purposes of the faking factor, we report a two-factor CFA with $g c$ in the supplementary material (SM Figure 2), which shows a correlation between the factor of faking and $g c$ of $r=.41$ (similar to results found by Geiger et al., 2018).

\section{Statistical Analysis}

The main analyses were conducted within the framework of structural equation modeling (SEM) with R (R Core Team, 2018) and the R package lavaan (Rosseel, 2012). The models presented in this paper are based on indicators of mean familiarity ratings of reals and foils. In order to circumvent possible problematic distributions of the variables (i.e., nonnormally distributed indicators), the reported models relied on a robust maximum likelihood estimator where necessary. To reduce model complexity, we used item parcels as indicators after establishing the unidimensionality of the scales (Little et al., 2002). In more detail, we parceled the indicators for overclaiming, self-reported knowledge, and knowledge items heterogeneously so that each parcel was stratified across all broad content domains (Cole et al., 2016; Steger et al., 2019). Furthermore, the intelligence scales were parceled with equal average difficulty. We used full information maximum likelihood estimation under the assumption of missing at random (i.e., missingness due to program failures: less than $2 \%$ of cell entries were missing) to combine missing data and parameter estimation in a single step (Enders, 2010). The following fit indices were considered as indication for good model fit: CFI (Comparative Fit Index) $\geq .95$, RMSEA (Root Mean Square Error of Approximation $) \leq .06$, and the SRMR $($ Standardized Root Mean Square Residual $) \leq .08(\mathrm{Hu}$ $\&$ Bentler, 1999). Prior to modeling, all variables were $z$-standardized. To make all analyses transparent and reproducible, we provide all material necessary to replicate the main findings in an online repository: https://osf.io/4bnv5/ 


\section{Results}

In Table 2, we report means and standard deviations of the mean familiarity ratings of the parcels that equally include the broad knowledge domains (Steger et al., 2019). The range of the descriptive statistics of the obtained parcels are similar to the descriptive statistics of other OCQs used the literature (e.g., Dunlop et al., 2019; Hülür et al., 2011).

\section{Table 2}

Descriptive Statistics for Familiarity Ratings, Including the Means, Standard Deviations, Skews, and Kurtosis for Each Parcel.

\begin{tabular}{ccccccccc}
\hline & & \multicolumn{4}{c}{ reals } & \multicolumn{4}{c}{ foils } \\
\cline { 4 - 8 } Parcel & no. reals & no. foils & $M(S D)$ & Skew & Kurtosis & $M(S D)$ & Skew & Kurtosis \\
\hline 1 & 25 & 8 & $2.74(.56)$ & .20 & -.12 & $1.55(.43)$ & 1.33 & 2.38 \\
2 & 29 & 6 & $2.68(.50)$ & .29 & .19 & $1.70(.57)$ & 1.02 & 1.26 \\
3 & 24 & 6 & $2.67(.51)$ & .23 & -.25 & $1.60(.47)$ & 1.50 & 2.64 \\
4 & 22 & 5 & $2.62(.49)$ & .25 & -.17 & $1.40(.44)$ & 1.71 & 3.75 \\
5 & 21 & 4 & $2.88(.56)$ & .23 & -.05 & $1.47(.51)$ & 1.63 & 3.26 \\
\hline
\end{tabular}

\section{Measurement Models}

To test for unidimensionality of the scales, we first report the fit indices for all individual measurement models (see Table 3). For all measurement models, fit indices were above the aforementioned cut-off values, indicating good to excellent model fit. However, the RMSEA of the measurement model for self-reported knowledge (Model 2) was insufficient, while CFI and SRMR both indicated that model fit was good. This deviating result concerning model fit might be due to the fact that the RMSEA tends to be over-sensitive for models with low degrees of freedom (Kenny et al., 2015). For faking ability, the CFI was below the threshold, while the RMSEA and the SRMR indicated a good model fit. We do not report fit 
indices for the measurement models of honesty-humility and openness, as these models only contained three indicators and were therefore exactly identified.

Table 3

Measurement Models for Overclaiming, Self-Reported Knowledge, Crystallized and Fluid Intelligence, and Creativity.

\begin{tabular}{|c|c|c|c|c|c|c|c|c|c|}
\hline \multicolumn{2}{|c|}{$\begin{array}{l}\text { Measurement } \\
\text { Model }\end{array}$} & \multirow{2}{*}{$\frac{n \text { (Parcels) }}{5}$} & \multirow{2}{*}{$\begin{array}{c}n \text { (Persons) } \\
297\end{array}$} & \multirow{2}{*}{$\frac{\chi^{2}}{4.70}$} & \multirow{2}{*}{$\frac{d f}{5}$} & \multirow{2}{*}{$\begin{array}{l}\text { CFI } \\
1.00\end{array}$} & \multirow{2}{*}{$\begin{array}{l}\text { RMSEA }[90 \% \mathrm{CI}] \\
.000[.000 ; .067]\end{array}$} & \multirow{2}{*}{$\begin{array}{c}\text { SRMR } \\
.012\end{array}$} & \multirow{2}{*}{$\begin{array}{l}\omega \\
.90\end{array}$} \\
\hline 1 & $\mathrm{OC}$ & & & & & & & & \\
\hline 2 & SRK & 5 & 297 & 14.19 & 5 & .994 & $.079[.034 ; .127]$ & .007 & .97 \\
\hline 3 & Gc & 5 & 293 & 6.34 & 5 & .996 & $.030[.000 ; .091]$ & .021 & .78 \\
\hline 4 & Gff / Gfv & 8 & 297 & 18.48 & 19 & 1.00 & $.000[.000 ; .050]$ & .028 & $.71 / .69$ \\
\hline 5 & $\begin{array}{l}\text { Faking } \\
\text { Ability }\end{array}$ & 5 & 295 & 8.37 & 5 & .936 & $.048 \quad[.000 ; .103]$ & .032 & .46 \\
\hline 6 & $\begin{array}{c}\text { DT / } \\
\text { Originality* }\end{array}$ & 6 & 298 & 5.72 & 8 & 1.00 & $.000[.000 ; .056]$ & .017 & $.77 / .28$ \\
\hline
\end{tabular}

In addition to the unidimensionality of the scales, we report a latent regression model, where overclaiming (i.e., a latent variable capturing the communality of five mean familiarity rating parcels for foils) predicts self-reported knowledge (i.e., a latent variable capturing the communality of five mean familiarity rating parcels for reals), as this model of the OCQ is the basis for all subsequent models (Figure 1; see SM Figure 3 for unstandardized parameters). We have selected a model in which overclaiming predicts self-reported knowledge.

Overclaiming accounted for $50.5 \%$ of the variance in self-reported knowledge in our sample.

\section{Figure 1}

Structural Model of Overclaiming (OC) and Self-Reported Knowledge (SRK) 


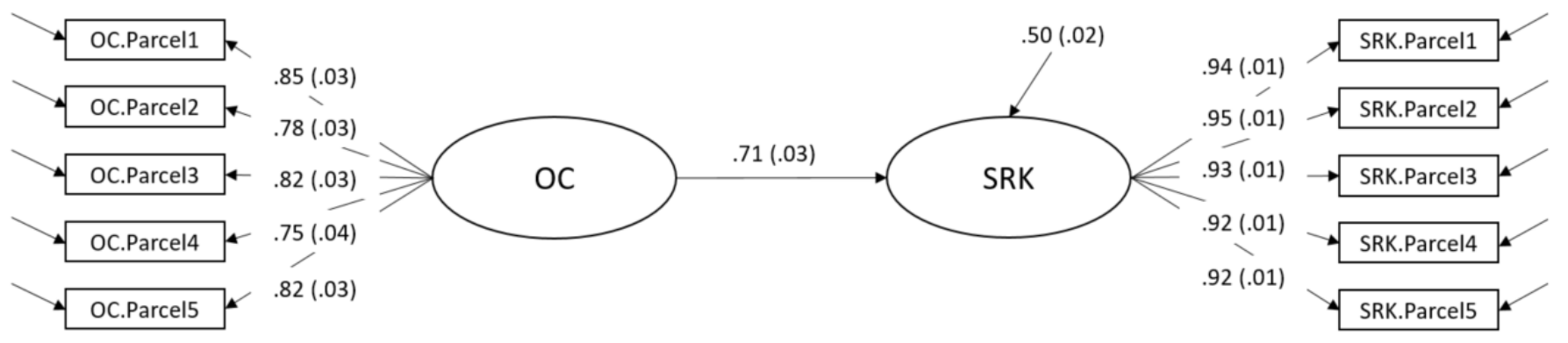

Note. Structural model of overclaiming (OC) and self-reported knowledge (SRK). All parameters are standardized. $n=298 ; \chi^{2}(34)=90.87, \mathrm{CFI}=.978, \mathrm{RMSEA}=.075$, SRMR $=.038$. The model including its unstandardized parameter estimates can be found in the supplementary material (SM Figure 3).

\section{Overclaiming as a Self-Enhancement Tendency}

In line with the first perspective, that overclaiming is a manifestation of an overarching tendency to self-enhance, overclaiming and self-reported knowledge should be predicted by self-reported honesty-humility, as well as the ability to fake (Figure 2, see SM Figure 4 for unstandardized parameters). However, honesty-humility did neither significantly predict self-reported knowledge nor overclaiming, whereas faking predicted self-reported knowledge, but not overclaiming. Thus, individual differences in honesty-humility and faking did not explain interindividual differences in overclaiming $\left(R^{2}<1 \%\right)$. For self-reported knowledge, a total of $58 \%$ in the variance was explained by all three predictors, however, it was only partly driven by the individual differences in faking ability. Given the empirical overlap between faking ability and $g c$ (e.g., Geiger et al., 2018), the explanatory power of faking ability for incrementally predicting self-reported knowledge is vanishingly low: extending the model with a factor of $g c$, the explanatory power of faking ability for selfreported knowledge disappears $\left(\beta=.06 ; \chi^{2}(220)=332.45, \mathrm{CFI}=.969, \mathrm{RMSEA}=.041\right.$, $\operatorname{SRMR}=.049)$.

\section{Figure 2}


Structural Model of Overclaiming (OC), Self-Reported Knowledge (SRK), Honesty-Humility, and Faking Ability

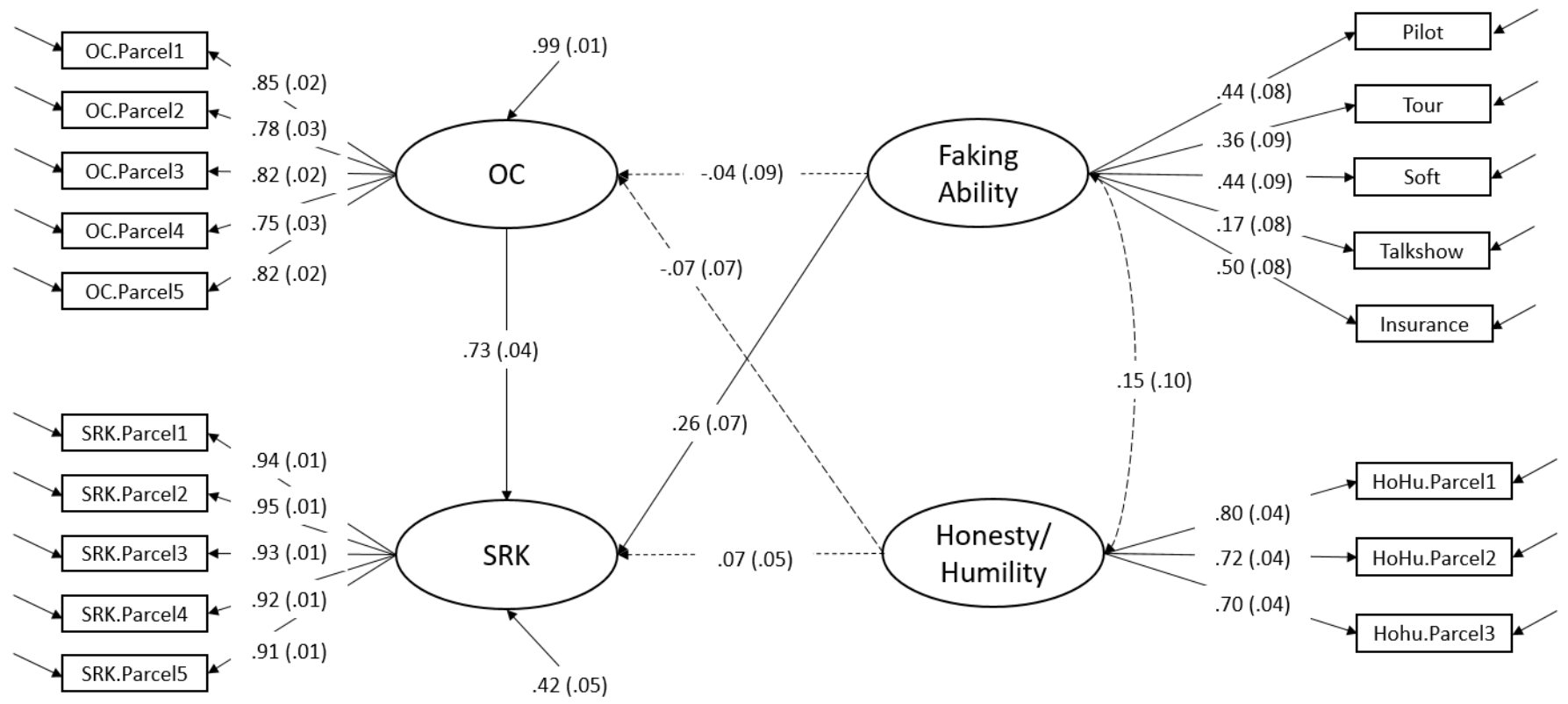

Note. Structural model of overclaiming (OC), self-reported knowledge (SRK), honestyhumility, and faking ability. All parameters are standardized; non-significant coefficients are displayed with dashed lines $(p<.05) . n=298 ; \chi^{2}(129)=215.64, \mathrm{CFI}=.972, \mathrm{RMSEA}=.047$, SRMR $=.049$. The model including its unstandardized parameter estimates can be found in the supplementary material (SM Figure 4).

\section{Overclaiming as an Index of Cognitive Ability}

Next, we juxtaposed overclaiming and self-reported knowledge with $g c$ and $g f$ to examine whether cognitive abilities account for the variance in overclaiming and self-reported knowledge (Figure 3, see SM Figure 5 for unstandardized parameters). Here, the variance of overclaiming was not explained by $g c$ or $g f$ at all, whereas $73 \%$ of the variance of selfreported knowledge was explained by overclaiming and $g c$. As expected, self-reported knowledge was largely predicted by overclaiming and only moderately predicted by actual $g c$. Although the regression weight between $g c$ and self-reported knowledge is in line with similar findings in the literature (e.g. Freund \& Kasten, 2012), these results generally and 
independent of the scoring approach discourage the use of overclaiming scales or self-report questionnaires as proxy measures of broadly defined cognitive abilities such as $g c$. However, because the OCQ can be transformed into simple word/non-word decision task, the mean familiarity ratings with reals correspond to a substantial degree with vocabulary tests (e.g., Ackerman and Ellingsen (2014) found a correlation of $r=.71$ between self-claimed vocabulary knowledge and a vocabulary score, and a correlation of $r=.43$ between self-rated knowledge and the false-alarm rate). The relations with $g f$ are so small that clearly selfreported knowledge cannot possibly serve as a proxy for $g f$.

\section{Figure 3}

Structural Model of Overclaiming (OC), Self-Reported Knowledge (SRK), and Knowledge $(G c)$, and Reasoning Ability $(G f)$

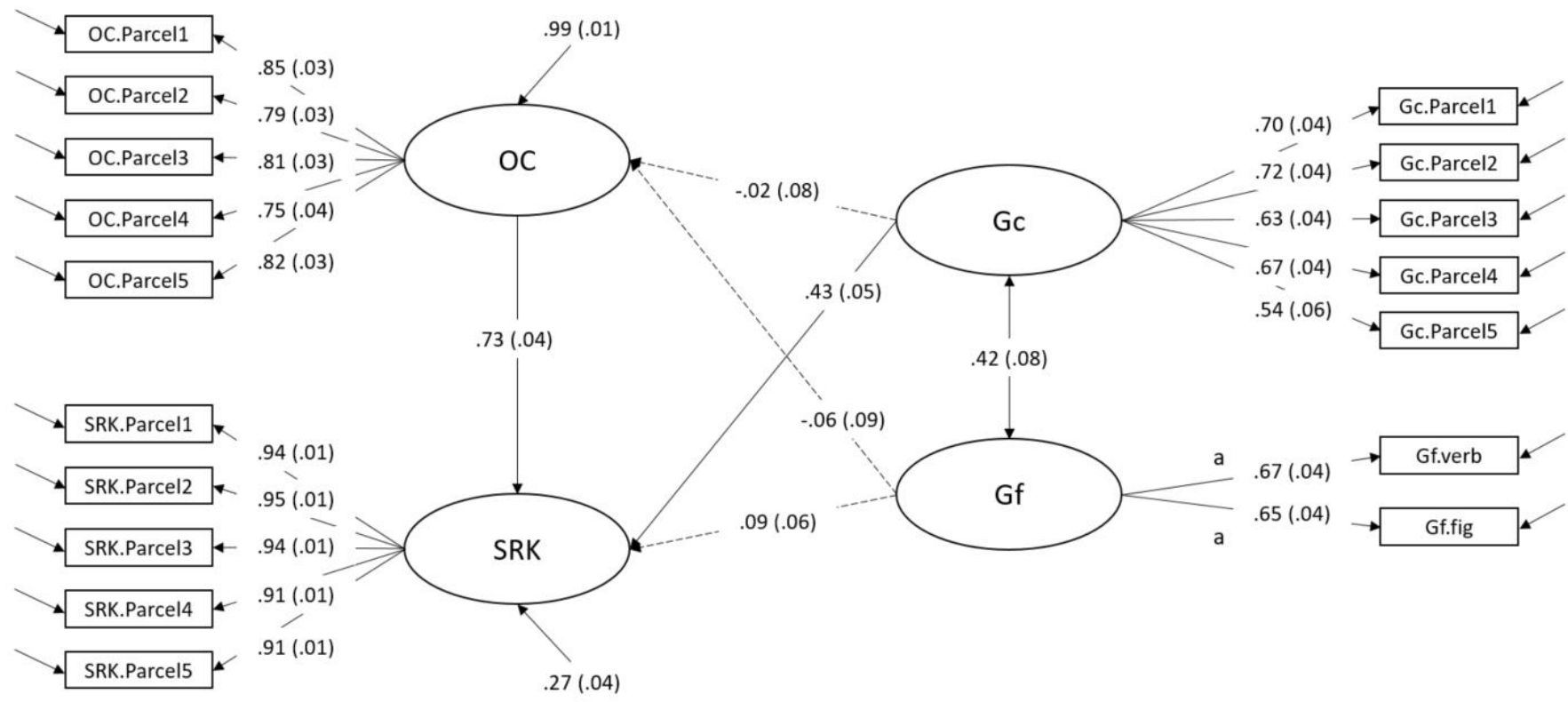

Note. All parameters are standardized; non-significant coefficients are displayed with dashed lines $(p<.05) . n=298 ; \chi^{2}(114)=199.78, \mathrm{CFI}=.975, \mathrm{RMSEA}=.050, \mathrm{SRMR}=.040$. The model including its unstandardized parameter estimates can be found in the supplementary material (SM Figure 5). 
Additionally, in the structural model modeling both $g c$ and $g f$, we tested whether $g c$ moderates the link between SRK and OC in a latent variable model. To do so, we first extracted the factor scores of $\mathrm{OC}$ and $g c$ in order to compute an interaction variable based on the product of the factor scores of OC and $g c$. This new variable was then entered into a latent regression model, where SRK was predicted by OC, $g c$, and the standardized interaction term. The model fitted the data: $\chi^{2}(102)=203.15, \mathrm{CFI}=.972, \mathrm{RMSEA}=.058, \mathrm{SRMR}=.048$. While the regression parameters were similar to the model above for the single predictors $\left(\beta_{\mathrm{OC}}=.72 ; \beta_{\mathrm{gc}}=.46, p<.001\right)$, the regression parameter of the interaction term was zero $\left(\beta_{\mathrm{OC}}^{* \mathrm{gc}}=.00, p=.99\right)$. This indicates that the association between SRK and OC is independent from the real knowledge of persons.

\section{Overclaiming as a Form of Creative Engagement}

Finally, we modeled overclaiming and self-reported knowledge together with openness-creativity, divergent thinking, and originality. According to this perspective, overclaiming is a byproduct of creative engagement. In this model, overclaiming and selfreported knowledge were predicted by divergent thinking, originality, and a factor for creativity self-reports from the openness facet of the HEXACO (Figure 4, see SM Figure 6 for unstandardized parameters). Overclaiming was significantly predicted by openness-creativity and self-reported knowledge was significantly predicted by divergent thinking. The predictors of creativity and openness were able to explain $10 \%$ of the variance of overclaiming and a total of $59 \%$ of the variance of self-reported knowledge was explained by all predictors.

\section{Figure 4}

Structural Model of Overclaiming (OC), Self-Reported Knowledge (SRK), and Divergent Thinking (DT), Originality, and Openness 


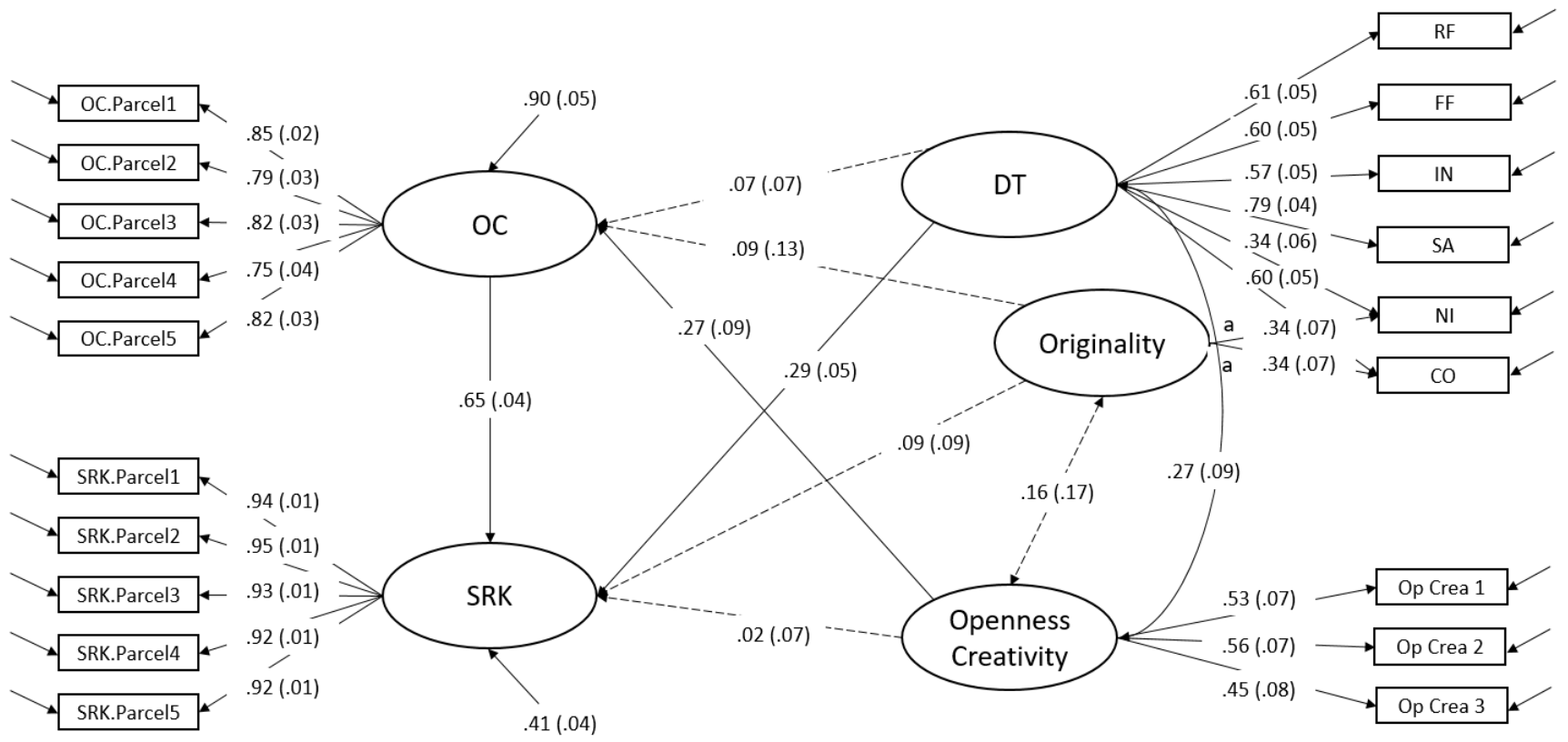

Note $. \mathrm{RF}=$ Retrieval Fluency, $\mathrm{FF}=$ Figural Fluency, $\mathrm{IN}=$ Inventing Names, $\mathrm{SA}=$ Similar Attributes, $\mathrm{NI}=$ Nicknames, $\mathrm{CO}=$ Combining Objects. All parameters are standardized; nonsignificant coefficients are displayed with dashed lines $(p<.05) . n=298 ; \chi^{2}(142)=225.38$, $\mathrm{CFI}=.975, \mathrm{RMSEA}=.044, \mathrm{SRMR}=.044$. The model including its unstandardized parameter estimates can be found in the supplementary material (SM Figure 6).

\section{Discussion}

The concept of overclaiming as a disposition is still vague and previous interpretations vary broadly, although there is some agreement in the literature as to the potential use of OCQs due to their efficient measurement properties (Dunlop et al., 2019). For our study, we distinguished between two fractions of an OCQ, namely, overclaiming (that is, mean familiarity ratings of foils) and self-reported knowledge (that is, mean familiarity ratings of reals). We linked both constructs with four perspectives on of what responses on OCQs might be reflective: self-enhancement tendencies (Paulhus et al., 2003), a cognitive processing bias due to the plausibility of foils (Müller \& Moshagen, 2018; Paulhus, 2011), a "practical measure of global cognitive ability" (Paulhus \& Harms, 2004, p. 311), and a form of creative engagement. The purpose of the present study was to critically test these interpretations and to 
strengthen the evidence with respect to the relation between overclaiming, self-reported knowledge, openness, and creative abilities. To understand individual differences in overclaiming and their antecedents, covariates, and consequences, we compiled an OCQ out of an existing measurement and newly-developed items that covers broad areas of knowledge and embedded overclaiming in the nomological net of cognitive abilities ( $g c$ and $g f$ ), personality (honesty-humility), faking ability, and creative abilities (divergent thinking and originality) and openness-creativity. Next, we discuss our results considering a scaffold of different interpretations of overclaiming behavior and address their implications.

\section{Overclaiming as a self-enhancement tendency}

The first perspective pictures overclaiming as a self-enhancement motivation (Paulhus et al., 2003), that is, describing oneself (deliberately) in a more positive manner. The urge to fake good (Ziegler, 2011) and therefore positively misrepresent oneself might be one of the possible explanations for self-enhancement (Bing et al., 2011; Feeney \& Goffin, 2015; Kam et al., 2015), as well as the personality factor of honesty-humility. From this perspective, a person claims to have knowledge that they actually do not have (Paulhus, 1984; Paulhus \& Reid, 1991) to appear more positive. Hence, we predicted that overclaiming should be more salient in individuals who on the one hand score low in trait honesty-humility as self-serving presentation corresponds with low honesty-humility scores (de Vries et al., 2014), and on the other hand are good at faking (i.e., score high on faking ability). However, our results show that individual differences in overclaiming could neither be explained by honesty-humility scores, nor by the ability to fake. The result for the association between overclaiming and honesty-humility fits into the broad spectrum of research regarding this association so far (e.g., Dunlop et al., 2019; Müller \& Moshagen, 2019, Steger et al., 2020). Although this current null result provokes the endorsement of an interpretation of overclaiming that understands it as more as a mere self-enhancement reflection, we want to mention that our research design was conducted in a low stakes setting, after all. 


\section{Overclaiming as a cognitive bias}

Overclaiming has been seen as reflecting some sort of cognitive processing bias (Müller \& Moshagen, 2018; Paulhus, 2011), possibly leading to a familiarity experience with terms that is based on morphologic or syntactic characteristics, rather than on actual reflections of one's knowledge (or deliberate faking when reporting one's knowledge). In the present study, the foils used to assess overclaiming were designed to be dissimilar from existing words to prevent remedies between foils and reals from being present in the OCQwhile still being aware that the validity of an overclaiming measure highly depends on the quality of foils (Ackerman \& Ellingsen, 2014). Please note that within this approach, it is essential to distinguish between mean effects (i.e., all subjects are equally vulnerable to cognitive biases) and individual differences effects (i.e., measures that differ with respect to provoking cognitive biases are not perfectly related to each other and differ in their correlations with covariates). Obviously, one option to further test this theoretical stance in future studies is to manipulate morphologic and syntactic features of foils. Tricking participants into believing they know certain foils that mimic existing terms raises the question of why some participants are more prone to produce biased answers than others. With our present results, it is difficult to clearly support or refute this notion, but we are confident that future studies will more thoroughly investigate this idea.

\section{Overclaiming as a proxy for cognitive abilities}

In line with the literature, we again found that mean familiarity ratings of foils were unrelated with knowledge (Hülür et al., 2011; 2014). By embedding overclaiming into a nomological net of cognitive abilities, we were able to show again that overclaiming strongly predicts self-reported knowledge (Hülür et al., 2011; 2014), whereas gc moderately predicted self-reported knowledge. These findings are in line with previous meta-analytic findings (Freund \& Kasten, 2012), implying that the immediate self-report of knowledge in the form of rating one's familiarity with real words is biased and, arguably, of limited utility in an applied 
context. With the current data, we cannot support the idea that self-reported knowledge in terms of mean familiarity ratings offer an economical tool for indexing cognitive abilities, since self-reported knowledge is confounded by overclaiming and only moderately explained by actual knowledge. By and large, overclaiming and self-reported knowledge are akin to measures of self-reported typical behavior-a measurement approach with which constructs of maximal cognitive effort such as fluid or crystallized intelligence cannot be captured. We suggest that — especially in light of previous studies also reporting low or moderate associations between overclaiming, OC bias, self-reported knowledge, OC accuracy, and $g f$ and $g c$ (Hülür et al., 2011, 2014; Paulhus \& Harms, 2004)—overclaiming as a phenomenon has a more complex nature: if someone is knowledgeable, overclaiming might tell us more about their personality, as this person might overclaim intentionally in order to appear positively. In contrast to that, overclaiming might reflect something more akin to maximal performance if someone less capable authentically reports what he or she think they know. Correspondingly, future studies investigating overclaiming should carefully consider on how to approach the assessment of this phenomenon.

\section{Overclaiming as a form of creative engagement}

Finally, overclaiming behavior was also discussed as the result of intellectual and creative investment traits (Dunlop et al., 2017; Hülür et al., 2011, 2014). This theoretical stance originates from the idea that overclaiming might arise due to more creative persons letting their fantasy flow. According to this perspective, subjectively new and unknown concepts - such as uncommon words, phrases, or neologisms — attract engagement and elaboration from open-minded, creative people, making them more vulnerable to claim familiarity with foils. However, our results show that overclaiming is not substantially predicted by facets of creative ability (divergent thinking and originality). As participants with the ability to fluently produce divergent solutions to a given problem might more easily sense 
a familiarity to one of those words, they could also be more prone to overclaim. On the other hand, the results showed that overclaiming was significantly predicted by openness-creativity.

Please note that openness moderately predicted overclaiming. This result joins the ranks of the hitherto inconclusive literature on the relation between openness and overclaiming and corroborates the evidence that there indeed is a weak connection between being open towards the unknown and overclaiming one's level of knowledge. Given the only moderate magnitude of this relation, we infer trying to understand overclaiming exclusively in terms of openness is futile, but that an open personality can contribute to overclaiming behavior.

\section{Conclusion}

Taken together, the test development and the presented pattern of results, which are in line with the literature (e.g., Ackerman \& Ellingesen, 2014; Bing et al., 2011; Dunlop et al., 2017; Dunlop et al., 2019; Hülür et al., 2011; Paulhus et al., 2003), support that we developed a psychometrically-sound OCQ. In contrast to the prevalent signal detection scoring, we distinguish self-reported knowledge from overclaiming in terms of the mean familiarity ratings of reals and foils, which is a straightforward approach to assess and score overclaiming. Although a substantial amount of variance between overclaiming and selfreported knowledge was shared, according to our results, this shared variance cannot be attributed to a common underlying factor for both types of items (foils vs. reals). In our study, we applied confirmatory factor analysis and discussed four competing perspectives on overclaiming and its nature. Based on our multivariate study, we were able to test competing perspectives on overclaiming. However, the design of our study did not allow for direct testing of overclaiming as a cognitive processing bias, or for discussing overclaiming in light of various application contexts, such as high stakes settings.

In sum, we have organized the state of the literature into four competing theoretical accounts, applied a comprehensive test battery including a variety of covariates, and evaluated 
structural equation models designed to capture the competing theoretical perspectives. We have shown that a) overclaiming is neither predicted by honesty-humility nor faking ability and therefore reflects something different than mere self-enhancement tendencies; b) carefully constructed foils allow reliable measurement of overclaiming, which is not best taken as indicating a cognitive processing bias; c) overclaiming is not predicted by crystallized intelligence, is highly predictive of self-reported knowledge, and self-reported knowledge is hence not suitable as an index of or a proxy for cognitive ability (more specifically crystallized intelligence); and d) overclaiming is not predicted by divergent thinking and originality, and moderately predicted by self-reported openness for creativity, indicating that overclaiming does not reflect creative ability. In conclusion, we have shed light into the nomological net of overclaiming by showing that it is highly predictive of what we think we know (self-reported knowledge), and by showing that no single theoretical perspective offers an exhaustive explanation of overclaiming behavior. 


\section{References}

Ackerman, P. L., \& Ellingsen, V. J. (2014). Vocabulary overclaiming - A complete approach: Ability, personality, self-concept correlates, and gender differences. Intelligence, 46, 216-227. https://doi.org/10.1016/j.intell.2014.07.003

Amabile, T. M. (1982). Social psychology of creativity: A consensual assessment technique. Journal of Personality and Social Psychology, 43, 997-1013.

Ashton, M. C., \& Lee, K. (2001). A theoretical basis for the major dimensions of personality. European Journal of Personality, 15, 327-353. https://doi.org/10.1002/per.417

Ashton, M. C., \& Lee, K. (2009). The HEXACO-60: A short measure of the major dimensions of personality. Journal of Personality Assessment, 91, 340-345. https://doi.org/10.1080/00223890902935878

Atir, S., Rosenzweig, E., \& Dunning, D. (2015). When knowledge knows no bounds: Selfperceived expertise predicts claims of impossible knowledge. Psychological Science, 26, 1295-1303. https://doi.org/10.1177/0956797615588195

Bensch, D., Maaß, U., Greiff, S., Horstmann, K. T., \& Ziegler, M. (2019). The nature of faking: A homogeneous and predictable construct? Psychological Assessment, 31, 532-544. https://doi.org/10.1037/pas0000619

Bensch, D., Paulhus, D. L., Stankov, L., \& Ziegler, M. (2017). Teasing apart overclaiming, overconfidence, and socially desirable responding. Assessment, 351-363. https://doi.org/10.1177/1073191117700268

Bing, M. N., Kluemper, D., Kristl Davison, H., Taylor, S., \& Novicevic, M. (2011). Overclaiming as a measure of faking. Organizational Behavior and Human Decision Processes, 116, 148-162. https://doi.org/10.1016/j.obhdp.2011.05.006

Borman, W., Kubisiak, C., \& Schneider, R. (1999). Work Styles. In N. Peterson, M. Mumford, W. Borman, R. Jeanneret, \& E. Fleishman (Eds.), An Occupational 
Information System for the 21st Century: The Development of $O * N e t$, (pp. 213-226). American Psychological Association.

Carroll, J. B. (1993). Human cognitive abilities: A survey of factor-analytic studies. Cambridge University Press.

Cole, D. A., Perkins, C. E., \& Zelkowitz, R. L. (2016). Impact of homogeneous and heterogeneous parceling strategies when latent variables represent multidimensional constructs. Psychological Methods, 21, 164-174. https://doi.org/10.1037/met0000047

de Vries, R. E., Zettler, I., \& Hilbig, B. E. (2014). Rethinking trait conceptions of social desirability scales: Impression management as an expression of honesty-humility. Assessment, 21, 286-299. https://doi.org/10.1177/1073191113504619

DeCarlo, L. T. (2010). On the statistical and theoretical basis of signal detection theory and extensions: Unequal variance, random coefficient, and mixture models. Journal of Mathematical Psychology, 54, 304-313. https://doi.org/10.1016/j.jmp.2010.01.001

Dunlop, P. D., Bourdage, J. S., de Vries, R. E., Hilbig, B. E., Zettler, I., \& Ludeke, S. G. (2017). Openness to (reporting) experiences that one never had: Overclaiming as an outcome of the knowledge accumulated through a proclivity for cognitive and aesthetic exploration. Journal of Personality and Social Psychology, 113, 810-834. https://doi.org/10.1037/pspp0000110

Dunlop, P. D., Bourdage, J. S., de Vries, R. E., McNeill, I. M., Jorritsma, K., Orchard, M., Austen, T., Baines, T., \& Choe, W.-K. (2019). Liar! Liar! (when stakes are higher): Understanding how the overclaiming technique can be used to measure faking in personnel selection. Journal of Applied Psychology. https://doi.org/10.1037/ap10000463

Enders, C. K. (2010). Applied missing Data Analysis. Guilford Press. 
Feeney, J. R., \& Goffin, R. D. (2015). The Overclaiming Questionnaire: A good way to measure faking? Personality and Individual Differences, 82, 248-252. https://doi.org/10.1016/j.paid.2015.03.038

Fell, C. B., König, C. J., Jung, S., Sorg, D., \& Ziegler, M. (2019). Are country level prevalences of rule violations associated with knowledge overclaiming among students?. International Journal of Psychology, 54, 17-22. https://doi.org/10.1002/ijop.12441

French, J. W., Ekstrom, R. B., \& Price, L. A. (1963). Manual for kit of reference tests for cognitive factors (revised 1963). Educational Testing Service.

Freund, P. A., \& Kasten, N. (2012). How smart do you think you are? A meta-analysis on the validity of self-estimates of cognitive ability. Psychological Bulletin, 138, 296-321. https://doi.org/10.1037/a0026556

Geiger, M., Olderbak, S., Sauter, R., \& Wilhelm, O. (2018). The "g" in faking: Doublethink the validity of personality self-report measures for applicant selection. Frontiers in Psychology, 9, 2153. https://doi.org/10.3389/fpsyg.2018.02153

Grosz, M. P., Lösch, T., \& Back, M. D. (2017). The narcissism-overclaiming link revisited. Journal of Research in Personality, 70, 134-138. https://doi.org/10.1016/j.jrp.2017.05.006

Hu, L., \& Bentler, P. M. (1999). Cutoff criteria for fit indexes in covariance structure analysis: Conventional criteria versus new alternatives. Structural Equation Modeling: A Multidisciplinary Journal, 6, 1-55. https://doi.org/10.1080/10705519909540118

Hülür, G., Wilhelm, O., \& Schipolowski, S. (2011). Prediction of self-reported knowledge with over-claiming, fluid and crystallized intelligence and typical intellectual engagement. Learning and Individual Differences, 21, 742-746. https://doi.org/10.1016/j.lindif.2011.09.006 
Hülür, G., Wilhelm, O., \& Schipolowski, S. (2014). Corrigendum to "Prediction of selfreported knowledge with over-claiming, fluid and crystallized intelligence and typical intellectual engagement” [Learning and Individual Differences 21 (6) (2011) 742746]. Learning and Individual Differences, 35, 153-154. https://doi.org/10.1016/j.lindif.2014.07.014

Jacobs, K. E., \& Roodenburg, J. (2014). The development and validation of the Self-Report Measure of Cognitive Abilities: A multitrait-multimethod study. Intelligence, 42, 521. https://doi.org/10.1016/j.intell.2013.09.004

Jäger, A. O., Süß, H.-M., \& Beauducel, A. (1997). Test für das Berliner IntelligenzStrukturmodell (BIS 4) [Berlin-Structure-Intelligence Test (BIS 4)]. Goettingen: Hogrefe.

Kam, C., Risavy, S. D., \& Perunovic, W. Q. E. (2015). Using over-claiming technique to probe social desirability ratings of personality items: A validity examination. Personality and Individual Differences, 74, 177-181. https://doi.org/10.1016/j.paid.2014.10.017

Kellen, D., \& Klauer, K. C. (2018). Elementary signal detection and threshold theory. In J. T. Wixted (Ed.), Stevens' Handbook of Experimental Psychology and Cognitive Neuroscience (Fourth Edition). John Wiley \& Sons.

Kenny, D. A., Kaniskan, B., \& McCoach, D. B. (2015). The performance of RMSEA in models with small degrees of freedom. Sociological Methods \& Research, 44, 486507. https://doi.org/10.1177/0049124114543236

Kruger, J., \& Dunning, D. (1999). Unskilled and unaware of it: How difficulties in recognizing one's own incompetence lead to inflated self-assessments. Journal of Personality and Social Psychology, 77, 1121-1134.

Little, T. D., Cunningham, W. A., Shahar, G., \& Widaman, K. F. (2002). To parcel or not to parcel: Exploring the question, weighing the merits. Structural Equation Modeling: A 
Multidisciplinary Journal, 9, 151-173.

https://doi.org/10.1207/S15328007SEM0902_1

Ludeke, S. G., \& Makransky, G. (2016). Does the over-claiming questionnaire measure overclaiming? Absent convergent validity in a large community sample. Psychological Assessment, 28, 765-774. https://doi.org/10.1037/pas0000211

Mabe, P. A., \& West, S. G. (1982). Validity of self-evaluation of abilities: A review and meta-analysis. Journal of Applied Psychology, 67, 280-296.

MacMillan, N. A., \& Creelman, C. D. (2005). Detection theory: A user's guide. Lawrence Earlbaum Associates Publishers.

McCrae, R. R., \& Costa, P. T. (1989). The Structure of Interpersonal Traits: Wiggins's circumplex and the five-factor model. Journal of Personality and Social Psychology, $56,586-595$.

Mesmer-Magnus, J., Viswesvaran, C., Deshpande, S., \& Joseph, J. (2006). Social desirability: The role of over-claiming, self-esteem, and emotional intelligence. Psychological Science, 336-356.

Mickes, L., Wixted, J. T., \& Wais, P. E. (2007). A direct test of the unequal-variance signal detection model of recognition memory. Psychonomic Bulletin \& Review, 14, 858865.

Moshagen, M., Hilbig, B. E., \& Zettler, I. (2014). Faktorenstruktur, psychometrische Eigenschaften und Messinvarianz der deutschsprachigen Version des 60-Item HEXACO Persönlichkeitsinventars [Factor structure, psychometric features and measurement invariance of the German version of the 60-item HEXACO personality inventory]. Diagnostica, 60, 86-97. https://doi.org/10.1026/0012-1924/a000112

Müller, S., \& Moshagen, M. (2018). Overclaiming shares processes with the hindsight bias. Personality and Individual Differences, 134, 298-300. https://doi.org/10.1016/j.paid.2018.06.035 
Müller, S., \& Moshagen, M. (2019). Controlling for response bias in self-ratings of personality: A comparison of impression management scales and the overclaiming technique. Journal of Personality Assessment, 101, 229-236. https://doi.org/10.1080/00223891.2018.1451870

Mussel, P. (2013). Intellect: A theoretical framework for personality traits related to intellectual achievements. Journal of Personality and Social Psychology, 104, 885906. https://doi.org/10.1037/a0031918

Paulhus, D. L. (1984). Two-component models of socially desirable responding. Journal of Personality and Social Psychology, 46, 598-609. http://dx.doi.org/10.1037/00223514.46.3.598

Paulhus, D. L. (2002). Socially desirable responding: The evolution of a construct. In H. I. Braun, D. N. Jackson, \& D. E. Wiley (Eds.), The role of constructs in psychological and educational measurement (pp. 49-69). Erlbaum Associates, Inc.

Paulhus, D. L. (2011). Overclaiming on personality questionnaires. In M. Ziegler, C. MacCann, \& R. D. Roberts (Eds.), New perspectives on faking in personality assessement (pp. 151-164). Oxford University Press, Inc.

Paulhus, D. L., \& Harms, P. D. (2004). Measuring cognitive ability with the overclaiming technique. Intelligence, 32, 297-314. https://doi.org/10.1016/j.intell.2004.02.001

Paulhus, D. L., Harms, P. D., Bruce, M. N., \& Lysy, D. C. (2003). The over-claiming technique: Measuring self-enhancement independent of ability. Journal of Personality and Social Psychology, 84, 890-904. https://doi.org/10.1037/0022-3514.84.4.890

Paulhus, D. L., \& Petrusic, W. M. (2007). Measuring individual differences with signal detection analysis: A guide to indices based on knowledge ratings.

Paulhus, D. L., \& Reid, D. B. (1991). Enhancement and denial in socially desirable responding. Journal of Personality and Social Psychology, 60, 307-317. 
Pennycook, G., \& Rand, D. G. (2019). Who falls for fake news? The roles of bullshit receptivity, overclaiming, familiarity, and analytic thinking. Journal of Personality, jopy.12476. https://doi.org/10.1111/jopy.12476

Phillips, D. L., \& Clancy, K. J. (1972). Some effects of "social desirability" in survey studies. American Journal of Sociology, 77, 921-940. https://doi.org/10.1086/225231

R Core Team. (2018). R: A language and environment for statistical computing (Version 3.5.1) [Computer software]. R Foundation for Statistical Computing. https://www.Rproject.org/

Randall, D. M., \& Fernandes, M. E. (1991). The social desirability response bias in ethics research. Journal of Business Ethics, 10, 805-817.

Raskin, R. N., \& Hall, C. S. (1979). A narcissistic personality inventory. Psychological Reports. http://dx.doi.org/10.2466/pr0.1979.45.2.590

Raubenheimer, A. S. (1925). An experimental study of some behavior traits of the potentially delinquent boy. Psychological Monographs, 34, 1-107.

Rosseel, Y. (2012). lavaan: An R Package for Structural Equation Moeling. Journal of Statistical Software, 48, 1-36.

Schoppe, K. J. (1975). Verbaler Kreativitäts-Test-VKT: ein Verfahren zur Erfassung verbalproduktiver Kreativitätsmerkmale [Verbal Creativity Test - VKT: A Procedure for Assessing Productive Creativity Features]. Verlag für Psychologie CJ Hogrefe.

Selker, R., van den Bergh, D., Criss, A. H., \& Wagenmakers, E.-J. (2019). Parsimonious estimation of signal detection models from confidence ratings. Behavior Research Methods, 51, 1953-1967.

Silvia, P. J., Kaufman, J. C., Reiter-Palmon, R., \& Wigert, B. (2011). Cantankerous creativity: Honesty-humility, agreeableness, and the HEXACO structure of creative achievement. Personality and Individual Differences, 51, 687-689. https://doi.org/10.1016/j.paid.2011.06.011 
Silvia, P. J., Winterstein, B. P., Willse, J. T., Barona, C. M., Cram, J. T., Hess, K. I., Martinez, J. L., \& Richard, C. A. (2008). Assessing creativity with divergent thinking tasks: Exploring the reliability and validity of new subjective scoring methods. Psychology of Aesthetics, Creativity, and the Arts, 2, 68-85. https://doi.org/10.1037/1931-3896.2.2.68

Stanislaw, H., \& Todorov, N. (1999). Calculation of signal detection theory measures. Behavior Research Methods, Instruments, \& Computers, 31, 137-149. https://doi.org/10.3758/BF03207704

Starns, J. J., \& Ratcliff, R. (2014). Validating the unequal-variance assumption in recognition memory using response time distributions instead of ROC functions: A diffusion model analysis. Journal of Memory and Language, 70, 36-52. https://doi.org/10.1016/j.jml.2013.09.005

Steger, D., Schroeders, U., \& Wilhelm, O. (2019). On the dimensionality of crystallized intelligence: A smartphone-based assessment. Intelligence, 72, 76-85.

Steger, D., Schroeders, U., \& Wilhelm, O. (2020). Caught in the act: Predicting cheating in unproctored knowledge assessment. Assessment.

Swets, J. A. (1986). Form of empirical ROCs in discrimination and diagnostic tasks: Implications for theory and measurement of performance. Psychological Bulletin, 99, 181-198.

Van Vaerenbergh, Y., \& Thomas, T. D. (2013). Response styles in survey research: A literature review of antecedents, consequences, and remedies. International Journal of Public Opinion Research, 25, 195-217. https://doi.org/10.1093/ijpor/eds021

Weiss, S., Steger, D., Kaur, Y., Hildebrandt, A., Schroeders, U., \& Wilhelm, O. (2020). On the trail of creativity: Dimensionality of divergent thinking and its relation with cognitive abilities and personality. European Journal of Personality. 
Weng, L.-J. (2004). Impact of the number of response categories and anchor labels on coefficient alpha and test-retest reliability. Educational and Psychological Measurement, 64, 956-972. https://doi.org/10.1177/0013164404268674

Wilhelm, O., Schroeders, U., \& Schipolowski, S. (2014). Berliner Test zur Erfassung fluider und kristalliner Intelligenz für die 8. Bis 10. Jahrgangsstufe (BEFKI 8-10) [Berlin test for the assessment of fluid and crystallized intelligence for grades 8-10 (BEFKI 810)]. Hogrefe.

Williams, K. M., Paulhus, D. L., \& Nathanson, C. (2002). The nature of overclaiming: Personality and cognitive factors. Poster presented at the 110th annual meeting of the American Psychological Association, Chicago, IL.

Wixted, J. T. (2020). The forgotten history of signal detection theory. Journal of Experimental Psychology: Learning, Memory, and Cognition, 46, 201-233. https://doi.org/10.1037/xlm0000732

Ziegler, M. (2011). Applicant faking: A look into the black box. Psychologist, 49, 29-36.

Ziegler, M. (2015). “F*** you, I won't do what you told me!”- Response Biases as Threats to Psychological Assessment. European Journal of Psychological Assessment, 31, 153-158. https://doi.org/10.1027/1015-5759/a000292

Ziegler, M., Kemper, C., \& Rammstedt, B. (2013). The vocabulary and overclaiming test (VOC-T). Journal of Individual Differences, 34, 32-40. https://doi.org/10.1027/1614$0001 / \mathrm{a} 000093$ 